\title{
Multidisciplinary out-patient treatment with monitoring by telemedicine for a large-volume multifocal pyogenic liver abscess in times of the pandemic
}

\author{
Johannes J. Tebbe ${ }^{1}$ (이 | Nidhi-Su-Ann Kulamadayil-Heidenreich ${ }^{\mathbf{1}}$ | \\ Holger Heidenreich $^{1}$ | Hans-Georg Bongartz ${ }^{2}$ | Sandra Tebbe ${ }^{3}$
}

${ }^{1}$ Department of Gastroenterology and Infectious Disease, University Hospital OWL, Campus Lippe, University of Bielefeld, Bielefeld, Germany

${ }^{2}$ Family Practice (GP), Fritzlar, Germany

${ }^{3}$ Medical Specialist Practice, Kassel, Germany

\section{Correspondence}

Johannes J. Tebbe, Department of Gastroenterology and Infectious Disease, University Hospital OWL, Campus Lippe, University of Bielefeld, Röntgenstrasse 18, 32756 Detmold, Bielefeld, Germany.

Email: johannes-josef.tebbe@klinikumlippe.de

\begin{abstract}
Pyogenic liver abscesses represent one of the rarer, but potentially life-threatening diseases of the liver. The treatment for large-volume liver abscesses is usually multimodal with percutaneous drainage combined with several days of treatment in hospital. We are presenting a report on a male patient with type- 2 diabetes mellitus who suffered from a multifocal liver abscess $(>10 \mathrm{~cm})$. Due to the exceptional situation caused by the corona pandemic, the patient was treated conservatively with non-standard treatment which involved a multidisciplinary team and out-patient visits. Follow-up to ensure the treatment would be successful was carried through dialogue with the GP responsible for the patient's care, as well as daily telemedicine visits. The daily telemedicine visits were supplemented by episodic follow-up testing of laboratory values and contrast-enhanced ultrasound scans (CEUS) of the liver. We show that purely conservative therapy can be successful in a case with a high risk of mortality by using a combination of close telemedical monitoring and proactive interdisciplinary collaboration with the GP.
\end{abstract}

\section{K E Y W O R D S}

CEUS, pyogenic liver abscess, telemedicine

\section{1 | INTRODUCTION}

Pyogenic liver abscesses are considered to be a rare, but life-threatening disease of the liver with a worldwide incidence of 2.3-17.6 cases per 100,000. ${ }^{1}$ For the past few years, however, the incidence has been increasing. Pyogenic liver abscesses now represent $50 \%$ of all abscesses in the abdominal cavity. ${ }^{2}$ The mortality of pyogenic liver abscesses has been reported in the literature at $11-31 \%{ }^{3,4}$ While the major causes of pyogenic liver abscesses were once ascribed to intra-abdominal infections (eg, appendicitis) or trauma of the hepatobiliary system, more than $55 \%$ of cases are now associated with an unknown cause of the disease. $^{3}$ The predominant bacterial spectrum includes Escherichia coli, Streptococcus spp., and anaerobes. ${ }^{4}$ In recent times, however, Klebsiella pneumoniae is increasingly being found in abscess biopsy samples, particularly in Asia. ${ }^{5,6}$ Affected patients often present with co-morbidities or risk factors, such as diabetes mellitus. ${ }^{7,8}$ Treatment generally involves in-patient admission, using a combination of antibiotic treatment and percutaneous drainage. ${ }^{9-11}$ Current guidelines recommend intravenous antibiotic 
treatment for up to 3 weeks, followed by oral antibiotics for 1-2 months. ${ }^{8,12,13}$ The diameter of the inflammatory liver lesion has significance for both the prognosis and the choice of treatment. Liver abscesses with a diameter of up to $5 \mathrm{~cm}$ can be treated conservatively using antibiotics alone with a very good prognosis; larger abscesses, by contrast, should be additionally treated using percutaneous drainage or surgery. Mortality increases with increasing size of the abscess and with decreasing Hb values. ${ }^{14,15}$

\section{CASE}

A 50-year-old male patient was referred to our clinic with the diagnosis of "liver tumor of unclear type". On examination, the patient was obese (115kg; $187 \mathrm{~cm}$; BMI: 32.9) and had presented to the doctor due to a deterioration in his condition. He reported complaints of $12 \mathrm{~kg}$ weight loss over the previous 4 weeks as well as increasing physical weakness and joint complaints. The patient had recorded fever spikes recurring in the evening approximately every 5 days, reaching up to $39^{\circ} \mathrm{C}$. The general doctor treating the patient (his general practitioner) identified a large tumor-like structure in the liver on a diagnostic ultrasound scan and referred the patient to the clinic.

The admission report from the clinic describes an oriented patient in a poor general condition and with obese nutritional status. Clinically, pulmonary findings were normal with vesicular breathing sounds. No dyspnea, no cyanosis. Heart sounds were clear with no extra sounds. Abdomen was soft with no pain on percussion or deep palpation. No visible changes to the skin, and no jaundice. No abnormalities found on palpation of the abdominal wall, but obesity was noted. Pain in the area of the right shoulder. With the exception of arterial hypertension and obstructive sleep apnea syndrome, the patient denied any prior medical history. The patient denied taking any regular medication.

Laboratory analysis showed leucocytosis of $13.3 / \mathrm{nL}$ (4-9/nL). Further results were recorded: D-dimer value of $3.31 \mathrm{mg} / \mathrm{L} \mathrm{FEU}(<0.55 \mathrm{mg} / \mathrm{L} \mathrm{FEU})$; ferritin of $1.853 \mathrm{ng} /$ $\mathrm{mL}(21-360 \mathrm{ng} / \mathrm{mL})$; and CRP of $18.8 \mathrm{mg} / \mathrm{dL}(<0.5 \mathrm{mg} /$ dL) A case of normochromic normocytic anemia was identified, with a Hb value of $12 \mathrm{~g} / \mathrm{dL}$ (14-18 g/dL). Serum glucose levels were recorded at $276 \mathrm{mg} / \mathrm{dL}(70-115 \mathrm{mg} /$ $\mathrm{dL})$; HbA1c was $93 \mathrm{mmol} / \mathrm{mol} \mathrm{Hb}(20-42 \mathrm{mmol} / \mathrm{mol} \mathrm{Hb})$. (Table 1).

A thoracic X-ray did not reveal any abnormalities. Ultrasound of the liver showed a polycystic liver lesion in the left lobe measuring approx. $7 \mathrm{~cm}$ in diameter. A further liver lesion located close to the capsule and measuring approx. $3 \mathrm{~cm}$ in diameter could be demarcated in liver segment IV. Both lesions appeared on contrast-enhanced ultrasound scan (CEUS) as typical augmented ring enhancements in the arterial phase with a drop in contrast signal in the center of the lesion. In the portal-venous and late phases, the lesion borders again appeared hyperechoic to iso-echogenic with a consistent lack of contrast signal within the lesion (Figure 1). This contrast pattern is typical of a liver abscess. The findings were confirmed using triphasic, contrast-enhanced CT: description of the CT imaging included a polycystic liver lesion in the left lobe measuring $68 \mathrm{~mm}$ in diameter, and a liver lesion (segment IV) measuring $25 \mathrm{~mm}$ in diameter, with a suspected finding of liver abscesses. (Figure 1).

The liver lesion in the left lobe was selected as the reference lesion for assessment during follow-up.

Diagnostic testing in microbiology showed evidence of Streptococcus intermedius in both peripheral blood cultures and from the lesion itself. Resistance testing showed that the strain isolated was sensitive to all standard antibiotic agents.

We arrived at the summary diagnosis of a multifocal pyogenic liver abscess with evidence of $S$. intermedius in a patient suffering from type- 2 diabetes mellitus.

Due to evidence of $S$. intermedius as the causative pathogen for this pyogenic liver abscess, extensive diagnostic testing was carried out to localize any potential source (gastroscopy, colonoscopy, dental, and ENT examination). In summary, no source for the infection could be found. The only potential source for infection which could be identified by us on the basis of the available literature was the use of interdental brushes by the patient which he reported as part of his routine dental care. ${ }^{16}$

The treatment regime focused on two areas: antibiotic treatment for the pyogenic liver abscess and optimization of the diabetes-related metabolic condition in this patient with type-2 diabetes mellitus. Following results from the resistogram, intravenous antibiotic treatment was initially started with ampicillin/sulbactam ( $6 \mathrm{~g} / 3 \mathrm{~g}$ daily) for 5 days.

The type- 2 diabetes mellitus was treated during the acute phase of the illness (days 0-5) using insulin substitution alone. The patient was given advice with respect to his diabetes management, including training on selfmonitoring of blood sugars and application of insulin.

Due to the situation resulting from the SARS-CoV2 pandemic, the patient could not continue treatment on an in-patient basis beyond the fifth day. As a result, we followed up his continued treatment on an out-patient basis. We arranged for a daily telemedicine visit with the patient, as well as close monitoring and administration of medication by his general practitioner. Additionally, we arranged for recurrent out-patient check-ups for ultrasound assessment of the liver and follow-up determination of laboratory parameters. (Figure 1; Figure 2; Table 1) The general 


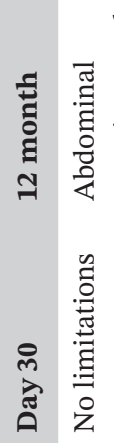

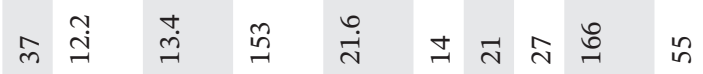

竞

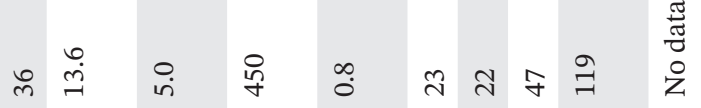

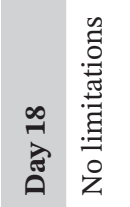

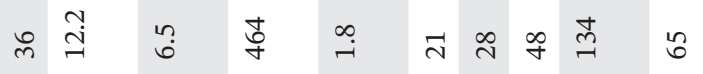

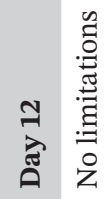

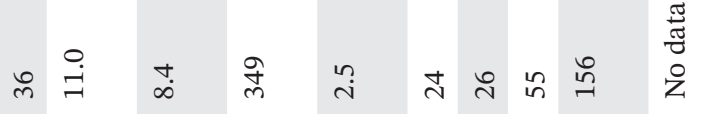

要

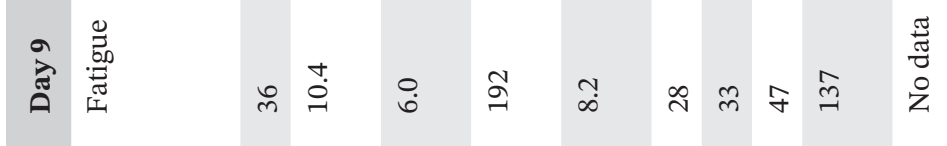

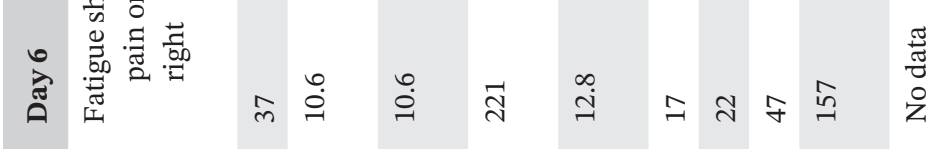

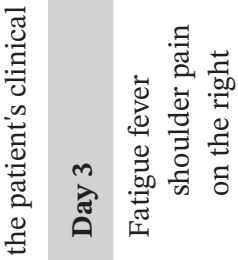

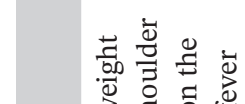

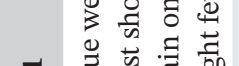

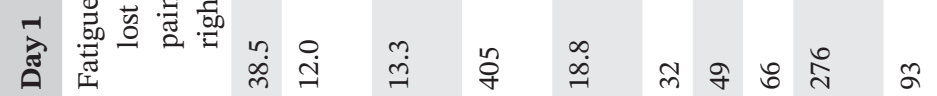

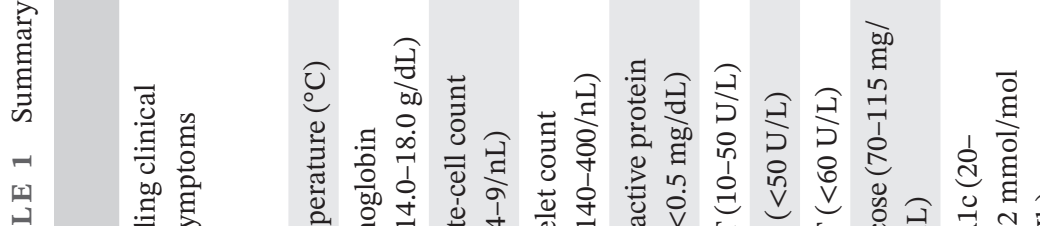

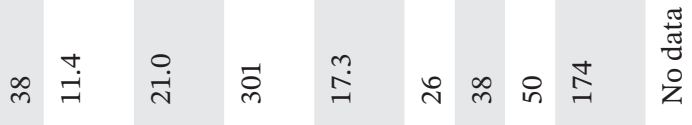



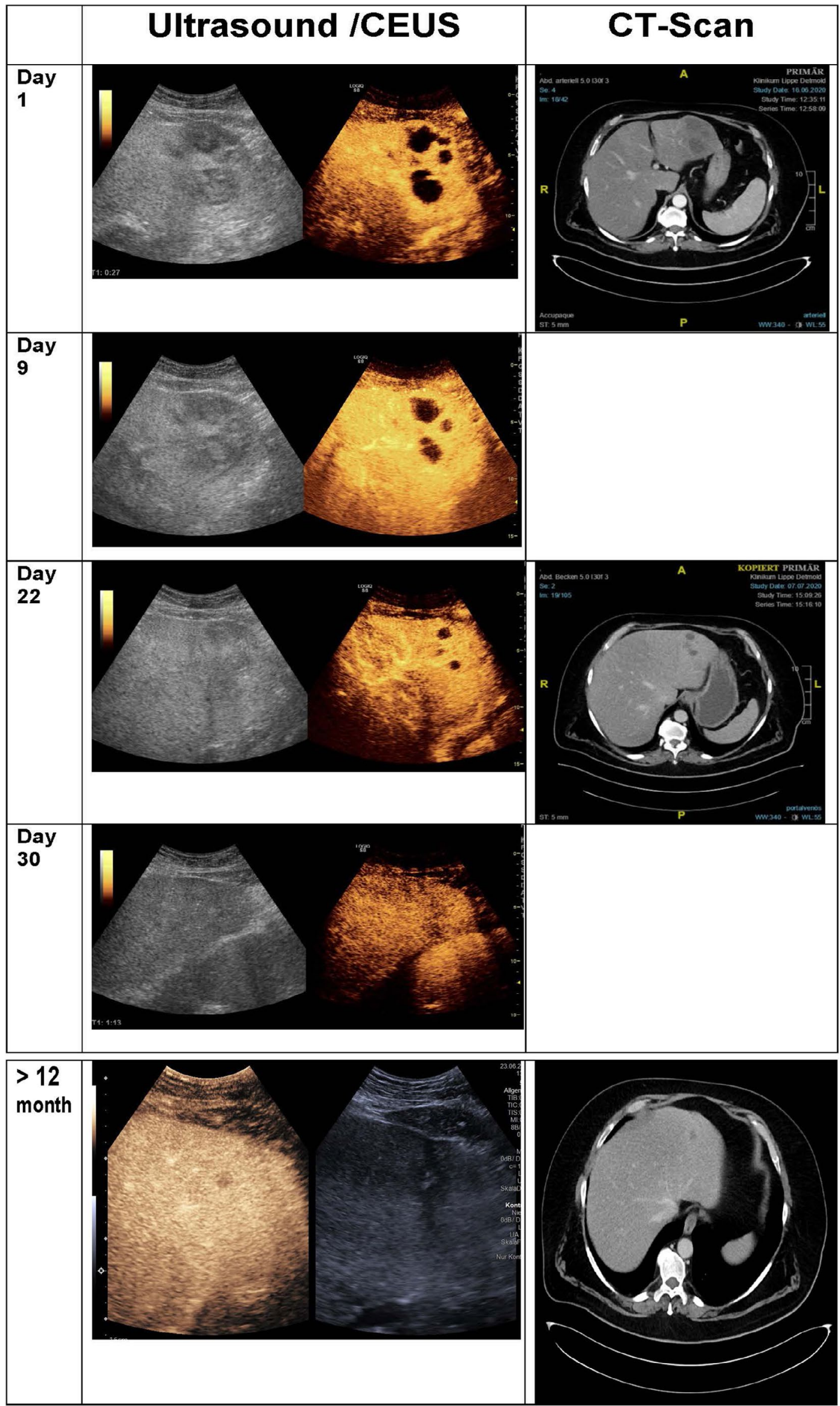

F I G U R E 1 Ultrasonography, CEUS, and CT scans in the course [days 1- 30 and 1 year after admission] 
FIGURE 2 Clinical course of laboratory parameters and medication. [black arrow $=$ start of insulin glargine; $\checkmark=$ start of metformin; 0 start of dulaglutide; $\boldsymbol{\Delta}$ = Parameters after 1 year]

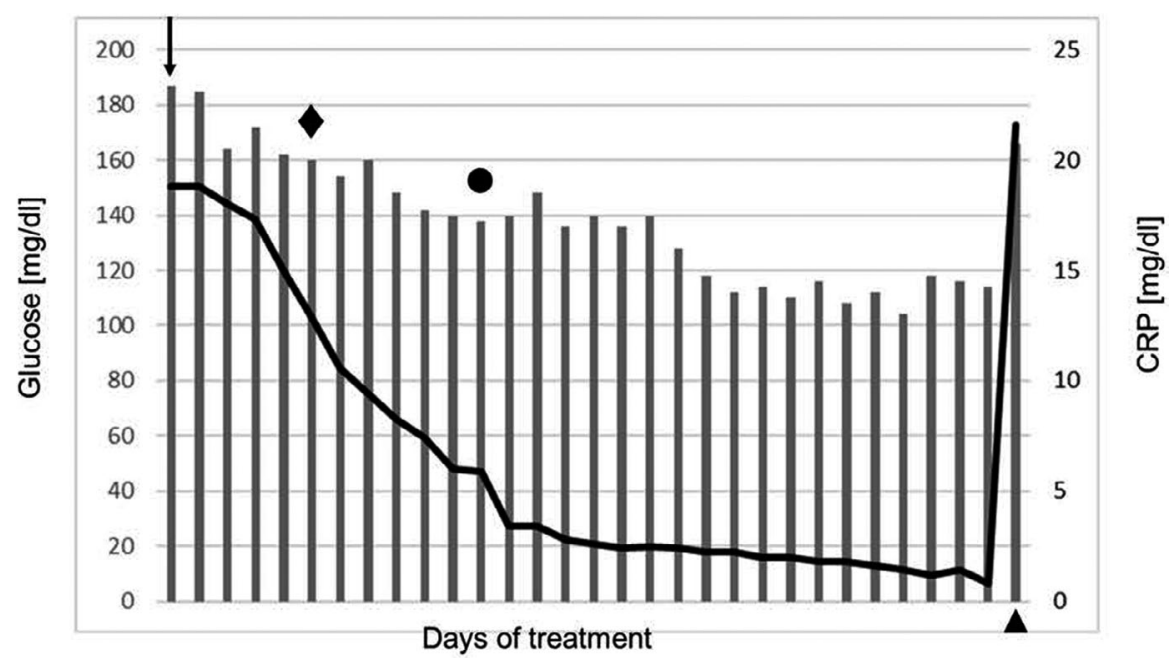

practitioner, supported by the specialist in the field of internal medicine, took over the daily telemedical rounds. These were supplemented by regular checks of the laboratory values. Monitoring of liver morphology by CEUS and CT scans was carried out by the specialist with the support of the clinic out-patient department.

During the twice-daily telemedicine visits (08:00 and 17:00), the patient was asked to provide values for body temperature, blood sugar, and body weight, as well as a general impression on how he was feeling. Out-patient check-ups with clinical diagnosis, laboratory analysis, and ultrasound examinations of the liver including CEUS were carried out at intervals of up to 14 days. The clinical visits were arranged working in close collaboration with the GP treating the patient. Medication was administered by the GP and adjusted as required.

The parameters over the course of recovery are outlined in Figure 2. During the out-patient check-ups, the medication-based treatments were continually adjusted with respect to the diabetes. On the sixth day of treatment, metformin was added to the treatment plan at a dose of $1 \mathrm{~g} 2 \mathrm{x}$ daily p.o. (1-0-1) due to a background of increasingly stable infective parameters. On the twelfth day, dulaglutide (1.5 mg 1x weekly) was added to the antidiabetic medications. At the same time, the insulin glargine dose was reduced by increments down to 12 IU taken at night. (Figure 2).

The intravenous antibiotic treatment was continued on an out-patient basis under the care of the GP. Substitution was continued for a further 10 days with amoxicillin/ clavulanic acid ( $875 \mathrm{mg} / 125 \mathrm{mg}$ ) 3x daily. On the 15th day of illness, the patient reported increasingly paste-like stool consistency. As a result, the antibiotic treatment was changed over to ceftriaxone (1x $2 \mathrm{~g}$ daily i.v.) in combination with a probiotic (E. Coli, nissle 1917). The stool sample taken at the time for diagnostic testing in microbiology did not produce any abnormal results. No evidence of clostridium difficile toxin A or B could be found. Treatment with ceftriaxone was continued for a further 14 days. Following this, no further antibiotic treatment was given.

On the 30th day of treatment, a follow-up CEUS of the liver was performed, as well as a CT abdomen. Imaging results at this time showed only rudimentary remnants of the large abscess in the left liver lobe (reference lesion), while the abscesses in the right liver lobe could no longer be identified. On the basis of the patient no longer having any complaints clinically, the almost normal laboratory values and the well-controlled diabetes mellitus, the close monitoring of this patient via telemedicine was ended at this time. (Figure 1; Table 1).

Almost 12 months following the first manifestation of the diabetes mellitus and the large-volume liver abscesses, the patient was re-admitted to the clinic on an in-patient basis due to complaints of new symptoms and increased infective parameters. The underlying cause on this occasion was acute appendicitis. An ultrasound scan of the liver (including CEUS) and a CT scan of the abdomen at this time only revealed small changes (most likely scartype alterations) in the left liver lobe with all other findings normal. (Figure 1; Table 1).

\section{3 | DISCUSSION}

Pyogenic liver abscesses represent one of the rarer, but potentially life-threatening diseases of the liver. ${ }^{1,12,17}$ In their randomized trial, Lee et al. showed that particular predictors for protracted hospital admissions were as follows: diabetes mellitus; arterial hypertension; positive identification of bacteria in culture; and reduced $\mathrm{Hb}$ values at the time of diagnosis. Independent risk factors identified as part of the trial also included an abscess diameter of $>5 \mathrm{~cm}$ and a significantly elevated CRP. ${ }^{15}$ 
If these factors were to be considered for the case we have presented here, then the initially poor prognosis for our case would have been associated with an indication for an in-patient hospital stay $>14$ days as well as surgical treatment for the liver abscess with percutaneous drainage. ${ }^{9,18}$ There are no data that recommend or consider purely conservative therapy possible for large or multifocal liver abscesses. All current data show that without doubt good therapy of all risk factors, such as diabetes mellitus, is crucial for the prognosis of the disease. ${ }^{19-22}$ This also applies to the case described here. However, the optimal therapy for diabetes mellitus can only be achieved through the cooperation of the patient and the close supervision by the GPs. This is also possible via telemedical offers. ${ }^{23,24}$ There has been no known use of telemedicine with respect to treatment monitoring for acute diseases of the liver. Experience has been described, however, of telemedicine approaches used in hepatology for the treatment and monitoring of patients with chronic liver disease, or following liver transplant. ${ }^{24,25}$ The 2020/2021 coronavirus pandemic has particularly broadened the application of telemedicine initiatives. ${ }^{26}$ There is also a long tradition of telemedicine applied to the treatment for diabetes mellitus: Experience has shown that telemedicine care applied to all forms of diabetes mellitus (types 1-3 and gestational diabetes) is qualitatively equal to regular care under a specialist, and can lead to good or very good long-term outcomes. ${ }^{27,28}$ In particular, continued surveillance of patients via telemedicine visits increases patient compliance in all cases. This has a significant impact on treatment success and certainly would have contributed to the outcome in the case presented by us here.

Our case also demonstrates how even rather banal irritations, such as over-zealous cleaning of teeth, may be sufficient to cause liver abscess in patients with untreated risk factors. This hypothesis is further reinforced by the fact that we isolated a typical organism in this respect: $S$. intermedius. The literature also describes case reports in this context for patients who had no known risk factors. ${ }^{16}$

Finally, our case report has shown how precise ultrasound follow-up of inflammatory liver lesions can be when using CEUS. The ultrasound imaging showed a good correlation with results from CT scans of the liver. (Figure 1) Furthermore, the regression of the lesion is a good indicator for evaluation of the effectiveness of medication-based anti-inflammatory treatments. ${ }^{29}$ This is also in line with the recommendations of the current guidelines for CEUS. ${ }^{30}$ CEUS is quick, easy-to-use, and is associated with very few side effects. As such, we would particularly like to encourage general practitioners to start using CEUS for specific indications as part of their portfolio of diagnostic tools.

\section{CONCLUSION}

We were able to demonstrate that the use of close monitoring via telemedicine and proactive interdisciplinary collaboration with the GP's involvement alongside conservative treatment meant a case with a high associated mortality risk could be transformed into a treatment success story. The total duration of treatment can even be shorter than for hospitalized patients on the basis of the literature. Both optimization of existing risk factors and a high level of flexibility in the treatment regime were the key to achieving success for treatment on an out-patient basis. Furthermore, we would like to use the experience from this case to point out the importance of ultrasound (including CEUS) for the diagnosis and surveillance of acute and chronic liver diseases.

\section{ACKNOWLEDGMENT}

None.

\section{CONFLICTS OF INTEREST}

The authors have declared that no conflict of interest exists.

\section{AUTHOR CONTRIBUTIONS}

All authors (JJT, NSAKH, HH, HGB, and ST) had full access to all of the data in the study and take responsibility for the integrity of the data and the accuracy of the data analysis. ST and JJT involved in concept and design, supervision, and drafting of the manuscript. NSAKH, HH, HGB, and JJT involved in acquisition, analysis, or interpretation of data. ST, NSAKH, HH, HGB, and JJT involved in critical revision of the manuscript for important intellectual content. NSAKH, HH, and HGB involved in administrative, technical, or material support.

\section{ETHICAL APPROVAL}

This case report was written in consensus with the patient concerned. All individual details in the manuscript were reviewed and approved by the patient prior to publication. The manuscript was published in accordance with the "European Medicines Agency Guidelines for Good Clinical Practice" and with written consent of the patient.

\section{DATA AVAILABILITY STATEMENT}

The data that support the findings of this study are available on request from the corresponding author. The data are not publicly available due to privacy or ethical restrictions.

\section{ORCID}

Johannes J. Tebbe (10) https://orcid.

org/0000-0001-5141-6813 


\section{REFERENCES}

1. Du ZQ, Zhang LN, Lu Q, et al. Clinical charateristics and outcome of pyogenic liver abscess with different size: 15-year experience from a single center. Sci Rep. 2016;6:35890.

2. Luo M, Yang X-X, Tan B, et al. Distribution of common pathogens in patients with pyogenic liver abscess in China: a meta-analysis. Eur J Clin Microbiol Infect Dis. 2016;35(10):1557-1565.

3. Pang TC, Fung T, Samra J, Hugh TJ, Smith RC. Pyogenic liver abscess: an audit of 10 years' experience. World J Gastroenterol. 2011;17(12):1622-1630.

4. Rahimian J, Wilson T, Oram V, Holzman RS. Pyogenic liver abscess: recent trends in etiology and mortality. Clin Infect Dis. 2004;39(11):1654-1659.

5. Qian Y, Wong CC, Lai S, et al. A retrospective study of pyogenic liver abscess focusing on Klebsiella pneumoniae as a primary pathogen in China from 1994 to 2015. Sci Rep. 2016;6:38587.

6. Zhang S, Zhang X, Wu Q, et al. Clinical, microbiological, and molecular epidemiological characteristics of Klebsiella pneumoniae-induced pyogenic liver abscess in southeastern China. Antimicrob Resist Infect Control. 2019;8:166.

7. Mukthinuthalapati V, Attar BM, Parra-Rodriguez L, Cabrera NL, Araujo T, Gandhi S. Risk factors, management, and outcomes of pyogenic liver abscess in a US safety net hospital. Dig Dis Sci. 2020;65(5):1529-1538.

8. Serraino C, Elia C, Bracco C, et al. Characteristics and management of pyogenic liver abscess: a European experience. Medicine (Baltimore). 2018;97(19):e0628.

9. Yu SC, Ho SS, Lau WY, et al. Treatment of pyogenic liver abscess: prospective randomized comparison of catheter drainage and needle aspiration. Hepatology. 2004;39(4):932-938.

10. Vogl TJ, Estifan F. Pyogenic liver abscess: interventional versus surgical therapy: technique, results and indications. Rofo. 2001;173(7):663-667.

11. Ferraioli G, Garlaschelli A, Zanaboni D, et al. Percutaneous and surgical treatment of pyogenic liver abscesses: observation over a 21-year period in 148 patients. Dig Liver Dis. 2008;40(8):690-696.

12. Chen YC, Lin CH, Chang SN, Shi ZY. Epidemiology and clinical outcome of pyogenic liver abscess: an analysis from the national health insurance research database of Taiwan, 20002011. J Microbiol Immunol Infect. 2016;49(5):646-653.

13. Giangiuli SE, Mueller SW, Jeffres MN. Transition to oral versus continued intravenous antibiotics for patients with pyogenic liver abscesses: a retrospective analysis. Pharmacotherapy. 2019;39(7):734-740.

14. He S, Yu J, Wang H, Chen X, He Z, Chen Y. Percutaneous fineneedle aspiration for pyogenic liver abscess $(3-6 \mathrm{~cm})$ : a twocenter retrospective study. BMC Infect Dis. 2020;20(1):516.

15. Lee $\mathrm{CH}$, Jo HG, Cho EY, et al. Maximal diameter of liver abscess independently predicts prolonged hospitalization and poor prognosis in patients with pyogenic liver abscess. BMC Infect Dis. 2021;21(1):171.

16. Livingston LV, Perez-Colon E. Streptococcus intermedius bacteremia and liver abscess following a routine dental cleaning. Case Rep Infect Dis. 2014;2014:954046.

17. Lardière-Deguelte $\mathrm{S}$, Ragot $\mathrm{E}$, Amroun $\mathrm{K}$, et al. Hepatic abscess diagnosis and management. J Visc Surg. 2015;152(4):231-243.
18. Mezhir JJ, Fong Y, Jacks LM, et al. Current management of pyogenic liver abscess: surgery is now second-line treatment. J Am Coll Surg. 2010;210(6):975-983.

19. Thomsen RW, Jepsen P, Sørensen HT. Diabetes mellitus and pyogenic liver abscess: risk and prognosis. Clin Infect Dis. 2007;44(9):1194-1201.

20. Li W, Chen H, Wu S, Peng J. A comparison of pyogenic liver abscess in patients with or without diabetes: a retrospective study of 246 cases. BMC Gastroenterol. 2018;18(1):144.

21. Du Z, Zhou X, Zhao J, et al. Effect of diabetes mellitus on shortterm prognosis of 227 pyogenic liver abscess patients after hospitalization. BMC Infect Dis. 2020;20(1):145.

22. Ko MC, Lin WH, Martini S, Chang YH, Chiu CT, Li CY. A cohort study of age and sex specific risk of pyogenic liver abscess incidence in patients with type 2 diabetes mellitus. Medicine (Baltimore). 2019;98(17):e15366.

23. Lee SWH, Chan CKY, Chua SS, Chaiyakunapruk N. Comparative effectiveness of telemedicine strategies on type 2 diabetes management: a systematic review and network metaanalysis. Sci Rep. 2017;7(1):12680.

24. Flodgren G, Rachas A, Farmer AJ, Inzitari M, Shepperd S. Interactive telemedicine: effects on professional practice and health care outcomes. Cochrane Database Syst Rev. 2015;2015(9):Cd002098.

25. Guarino M, Cossiga V, Fiorentino A, Pontillo G, Morisco F. Use of telemedicine for chronic liver disease at a single care center during the COVID-19 pandemic: prospective observational study. J Med Internet Res. 2020;22(9):e20874.

26. Serper M, Cubell AW, Deleener ME, et al. Telemedicine in liver disease and beyond: can the COVID-19 crisis lead to action? Hepatology. 2020;72(2):723-728.

27. Ming WK, Mackillop LH, Farmer AJ, et al. Telemedicine technologies for diabetes in pregnancy: a systematic review and meta-analysis. J Med Internet Res. 2016;18(11):e290.

28. Tchero H, Kangambega P, Briatte C, et al. Clinical effectiveness of telemedicine in diabetes mellitus: a meta-analysis of 42 randomized controlled trials. Telemed J E Health. 2019;25(7):569-583.

29. K C.S, Sharma D. Long-term follow-up of pyogenic liver abscess by ultrasound. Eur J Radiol. 2010;74(1):195-198.

30. Dietrich CF, Nolsøe CP, Barr RG, et al. Guidelines and good clinical practice recommendations for contrast-enhanced ultrasound (CEUS) in the liver-update 2020 WFUMB in cooperation with EFSUMB, AFSUMB, AIUM, and FLAUS. Ultrasound Med Biol. 2020;46(10):2579-2604.

How to cite this article: Tebbe JJ, KulamadayilHeidenreich N-S, Heidenreich H, Bongartz H-G, Tebbe S. Multidisciplinary out-patient treatment with monitoring by telemedicine for a large-volume multifocal pyogenic liver abscess in times of the pandemic. Clin Case Rep. 2021;9:e04970. https:// doi.org/10.1002/ccr3.4970 